\title{
Monte-Carlo analysis of challenges and limitations of dispersion-based optical thermometry
}

\author{
Anni Röse $^{1}$, Paul Köchert ${ }^{1}$, Günther Prellinger ${ }^{1}$, Eberhard Manske ${ }^{2}$ and Florian Pollinger ${ }^{1}$ \\ ${ }^{1}$ Physikalisch-Technische Bundesanstalt (PTB), Bundesallee 100, 38116 Braunschweig, \\ 2IImenau University of Technology, Max-Planck-Ring 14, 98693 IImenau \\ anni.roese@ptb.de
}

\begin{abstract}
Summary: Using the dispersion models, it is possible to derive the effective temperature in an optical beam by the measurement of the optical path length at two well-known optical wavelengths. We are currently developing a gradient thermometer based on this principle, deploying multi-wavelength interferometry for the length measurement. This shall enable beam bending compensation in optical levelling of large structures. A Monte-Carlo investigation reveals practical obstacles and limitations of this promising temperature measurement approach.
\end{abstract}

Keywords: dispersive thermometry, measurement uncertainty modelling, multi-wavelength interferometry, Monte-Carlo Simulation

\begin{abstract}
Motivation
When performing large-scale dimensional measurements outside, the measurement is mainly influenced by the inhomogeneity of the index of refraction. Vertical temperature gradients, e.g., induces beam bending. This limits the achievable accuracy of optical levelling. To improve the accuracy this effect must be properly compensated. But outdoor capturing of the temperature gradients by classical sensors requires dense and impractical sensor networks. Therefore, our group develops an alternative temperature sensor based on multi-wavelength absolute interferometry [1]. In this contribution, we present a Monte Carlo study of the achievable measurement uncertainty for this highly complex sensor design.
\end{abstract}

\section{Dispersive Thermometry}

The Edlén equation in the version by Bönsch und Potulski [2] describes the functional dependence of the refractive index of air by

$n\left(\lambda, T, \mathrm{p}, \mathrm{x}, \mathrm{p}_{\mathrm{w}}\right)=K(\lambda) \cdot D(\mathrm{~T}, \mathrm{p}, \mathrm{x})-p_{w} g(\lambda)$

with the wavelength $\lambda$ and the thermodynamic parameters pressure $\mathrm{p}$, temperature $\mathrm{T}$, carbondioxide contents $\mathrm{x}$, partial pressure of water vapor $p_{w}$, and the dispersion term $K(\lambda)$, the density term $D(\mathrm{~T}, \mathrm{p}, \mathrm{x})$, and the humidity term $g(\lambda)$ [2]. Dispersive thermometry is based on a measurement of the same geometric path $l$ with two different vacuum wavelengths $\lambda_{1}$ and $\lambda_{2}$. If performed in air, the optical pathlength will differ by their difference in refractivity.

$$
\begin{aligned}
& \mathrm{d}_{1}=\left(D K_{1}-\mathrm{p}_{\mathrm{w}} g_{1}\right) \times l+l \\
& \mathrm{~d}_{2}=\left(D K_{2}-\mathrm{p}_{\mathrm{w}} g_{2}\right) \times l+l
\end{aligned}
$$

This equation system can now be solved for the temperature (in ${ }^{\circ} \mathrm{C}$ ) including the humidity terms $K(\lambda), D(\mathrm{~T}, \mathrm{p}, \mathrm{x})$ and $g(\lambda)$ :

$\mathrm{T}_{\mathrm{eff}}=\frac{d_{2}-d_{1}+\left(K_{1}-K_{2}\right) l \mathrm{p} \alpha(1+\beta \mathrm{p})-\mathrm{p}_{\mathrm{w}} l\left(g_{1}-g_{2}\right)}{-\delta\left(d_{2}-d_{1}\right)+\alpha \mathrm{p}^{2} \gamma l\left(K_{1}-K_{2}\right)+\mathrm{p}_{\mathrm{w}} \delta l\left(g_{1}-g_{2}\right)}$

with $\beta, \gamma, \delta$ representing numerical constants and $\alpha$ a function of the $\mathrm{CO}_{2}$ contents $x$ [4].

The effective temperature along a beam path can hence be derived from the path $\left(d_{2}-d_{1}\right)$ if pressure and water vapor information is available from auxiliary sensors.

\section{Measurement and Uncertainty}

We measure the optical path lengths $d_{1}$ and $d_{2}$ using fixed synthetic wavelength interferometry [4]. We use two Nd:YAG laser with wavelengths $\lambda_{1}=532 \mathrm{~nm}$ and $\lambda_{2}=1064 \mathrm{~nm}$. The frequency difference $\Delta v_{i}$ between both lasers can be varied between $20 \mathrm{GHz}$ and $60 \mathrm{MHz}$. This corresponds to synthetic wavelengths between metres and millimetres. For the actual measurement, we detect the optical phases of all four fundamental optical beams and calculate the phase of the corresponding synthetic wavelengths. This measurement strategy, however, is very complex and suffers from highly unfavourable uncertainty scaling. Figure (1) shows an Ishikawa diagram which summarizes the various uncertainty contributions. Their individual impact as well as consequences on the instrument design can be better understood by a Monte Carlo simulation. 


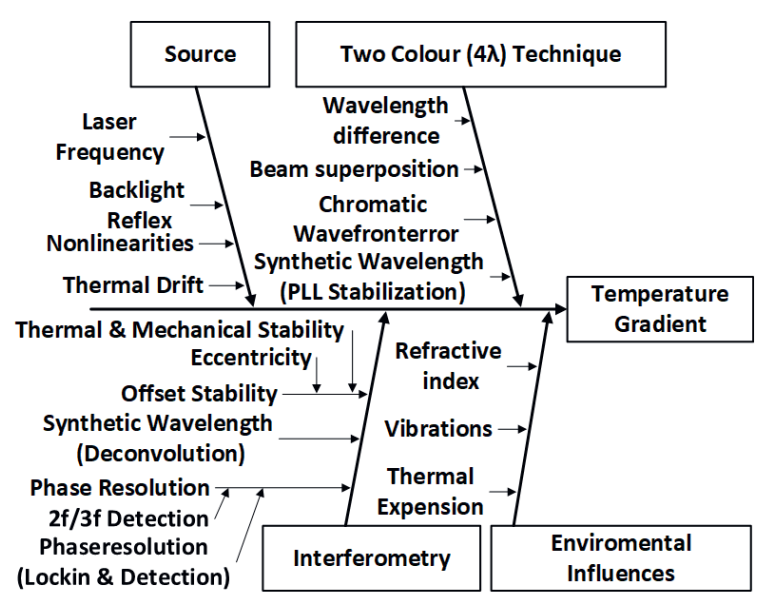

Figure 1: Ishikawa diagram summarizing uncertainty contributions of the dispersive thermometer.

\section{Results}

As discussed before, the measurement principle depends on an accurate measurement of the optical path difference. Any uncertainty of this measurement is unfavourably scaled up and impedes the achievable measurement uncertainty of the temperature measurement. As an example, Figure (2) shows the influence of thermal expansion of the reference path due to changes in temperature during the interference measurement. For an aluminium base plate with a thermal expansion of $23.1 \times 10^{-6} \mathrm{~K}^{-1}$, an interferometer reference path longer than $2 \mathrm{~mm}$ would make an uncertainty of $0.1 \mathrm{~K}$ very difficult to achieve.

It appears logical that the resolution of the optical phases is critical. But Figure (3) reveals how crucial this influence really is. For a measurement distance of $50 \mathrm{~m}$, and a synthetic wavelength $3.75 \mathrm{~mm}$, a resolution of the phase of at least $\pi / 10000$ is needed for a useful temperature uncertainty in the below $1 \mathrm{~K}$. Similarly, the Monte Carlo simulation shows that

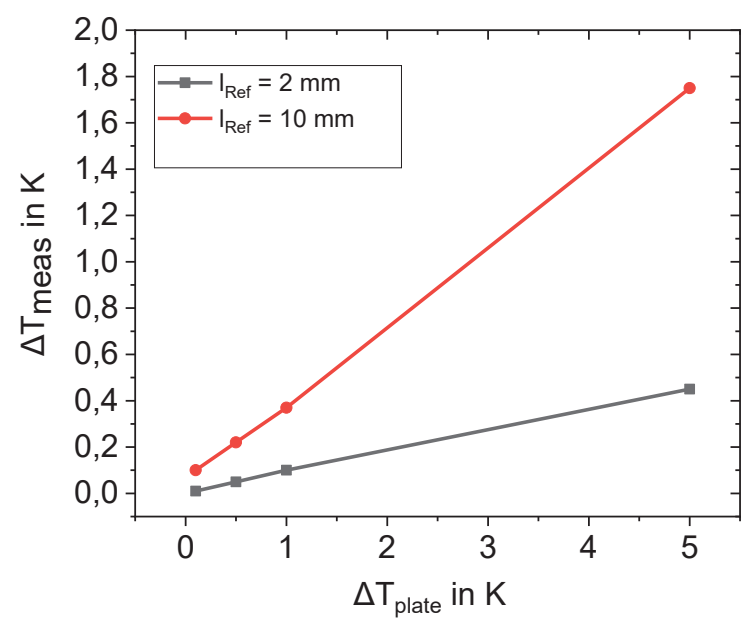

Figure 2: Thermal expansion base plate during the experiment with reference length between 2 $\mathrm{mm}$ and $10 \mathrm{~mm}$. for distances below $10 \mathrm{~m}$, the chosen setup with a synthetic wavelength of $20 \mathrm{GHz}$ is not capable to resolve temperatures better than $1 \mathrm{~K}$. For shorter distances, phase resolutions better than $\pi / 10000$ would be necessary.

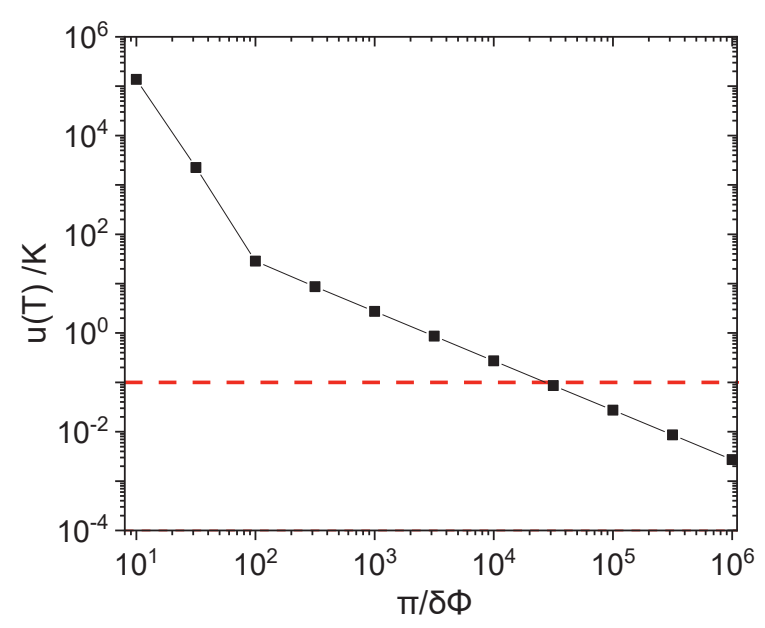

Figure 3: Dependence of the effective temperature uncertainty $u(T)$ (coverage factor $k=1$ ) on phase resolution $\delta \phi$.

\section{Conclusions}

In general, the phase resolution required by the Monte Carlo simulation poses a great challenge for the modulation and stabilization of the laser source, especially for an outdoor measurement. To achieve this, considerable integration times will probably be necessary, too. The lower limit of $10 \mathrm{~m}$ for this measurement is another interesting result, but less critical here since the targeted application, the reference point measurement of large VLBI telescopes, implies longer distances. In conclusion, this study shows that the realization of an optical thermometer for this purpose is, though challenging, feasible, with the Monte Carlo analysis pointing to critical issues for the instrument design.

\section{Acknowledgement}

This project has received funding from the EMPIR programme cofinanced by the participating States and from the European Union's Horizon 2020 research and innovation programme.

\section{References}

[1] Meiners-Hagen K, Meyer T, Prellinger G, Pöschel W, Dontsov D, and Pollinger F 2016 Opt. Expr. 2424092

[2] Bönsch G and Potulski E 1998 Metrologia 35 133

[3] Meiners-Hagen K, Schödel R, Pollinger F, and Abou-Zeid A 2009 Meas. Sci. Rev. 916

[4] Meiners-Hagen $K$ and Abou-Zeid A 2008 Meas. Sci. Technol. 19084004 